\title{
Constructing Pornographic Images Detector based on naïve Bayesian classifier
}

\author{
Alaa Y. Taqa \\ Department of Computer Science \\ College of Education \\ University of Mosul \\ alaa.taqa@gmail.com
}

Received

09 / 11 / 2008
Bayez K. AL-Sulaifanie

bayez_k@yahoo.com

Accepted

16 / 02 / 2009

\section{المستخلص}

لكتشف الصور الإبلحية على نحو فعل يمكن أن يمنع من ششر هذه الصور على

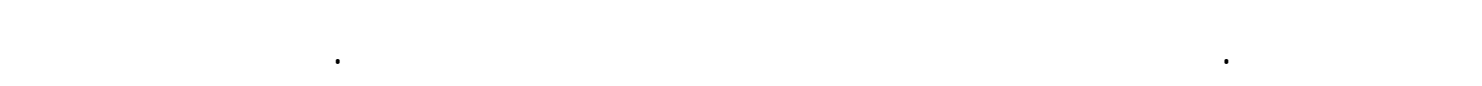

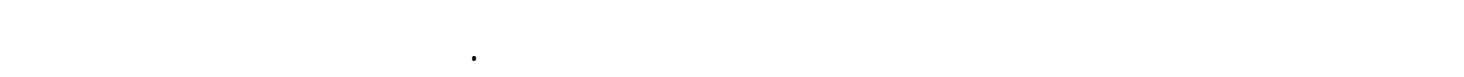

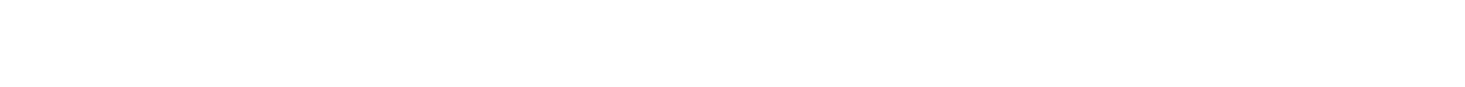

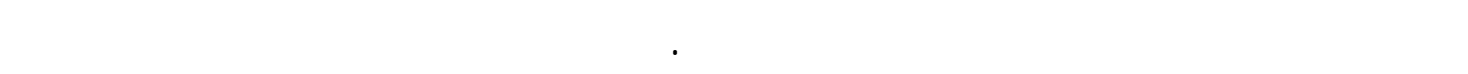

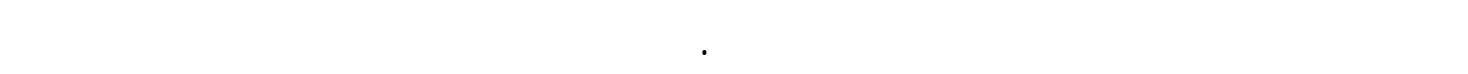

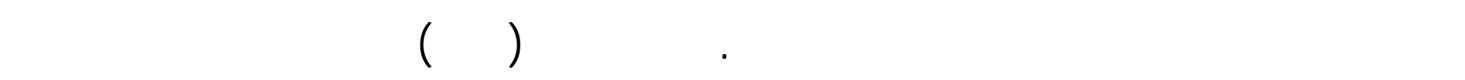

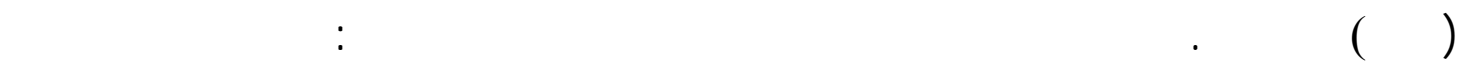

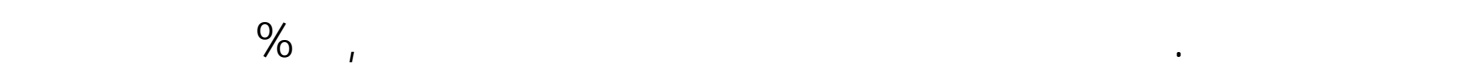

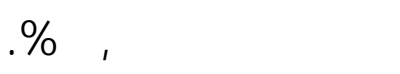

\section{Abstract}

Detection of pornographic images can effectively prevent pornographic images from spreading on the Internet. This research proposes a new approach of pornographic images detector. Naive Bayesian classifier is used by the proposed detector to identify potential pornographic images. Skin and non-skin color models are constructed and exploited by constructing a Bayesian decision rule based skin detector. Several features are extracted from the output of skin detector which forms the features vector. The naive Bayesian classifier is trained on these features for both porn and non-porn classes. An experiment used 
(136) images for training the pornographic images detector and (154) images for testing it. The pornographic images detector is evaluated by using sensitivity, precision, specificity and accuracy metrics. It achieves a detective rate of $(91.48 \%)$ with $(6.67 \%)$ false positive rate.

\section{Introduction}

Recent development of multimedia availability on the Internet has enabled Internet users around the world to access enormous amounts of video-, picture-, and audio-files. However, this has also led to new business opportunities for the adult -industry. Furthermore, due to this industry's aggressive marketing, it has become very difficult to avoid stepping a porn site from time to time, be it through web - surfing search results from a web search - engine. So, the detection of pornographic images can effectively prevent pornographic images from spreading on the Internet.

Many different approaches have been considered in the literature in order to detect pornographic images. Jones and Rehg [1] refer to an interesting application of skin detection which is part of a larger system for detecting people in photos. A person detector that worked reliably on Web images could be a valuable tool for image search services on the web and in digital libraries, as well as for image categorization. By taking advantage of the fact that there is a strong correlation between images with large patches of skin and pornographic images, the skin detector can also be used as the basis for image detector.

The first step in pornographic images detector is skin detection. The output of this detector is then used to extract features vector [2][3]. The final task is then to find the decision rule on this features vector that optimally detects pornographic images from those not [4][3].

The features vector is computed for each image in the training set. Then it is used to train a classifier on these features to determine whether a nudity or pornography for any given image is present or not [1].

The ANN adopted by Zheng et al.[2] takes the feature vector as input to MLP, and produces a real number $(x)$ as output, which can be compared to a threshold $(T)$ to decide whether the input vector color image is pornographic or not. Both Lin et al. [5] and Rowly et al. [6] use the features vector as the input of SVM, which is considered as an excellent tool for classification with learning ability.

This research proposes a new approach of pornographic images detector by using naïve Bayesian classifier for detecting and filtering the pornographic images; while the skin detector is constructed by using Bayesian decision rule based skin detector. 
This research is organized as follows: sections 2 explains the skin detection, and segmentation. Section 3 illustrates pornographic image detection. Section 4 presents the practical part in details. Finally, the conclusions and future work are discussed in section 5 .

\section{Skin Detection}

Skin detection can be defined as the process of selecting which pixels of a given image correspond to human skin [7].

The detection of skin regions in color images is a preliminary step in several application, such as face detection and pornographic images detection [8][4].

The main goal of skin color detection algorithm is to build a decision rule that will discriminate between skin and non-skin pixels. This is accomplished by introducing a metric, which measures distance between the pixel colors to skin tone. The type of this metric is defined by the skin color modeling method. Many different methods for discriminating between skin pixels and non-skin pixels are available in the literature. These can be grouped in three types of skin modeling: Parametric, non-parametric and explicit skin cluster definition methods.

The explicit skin cluster classifier defines experimentally the boundaries of the skin cluster in certain color space [9].

Zheng et al. [4] suggest using statistical approach in skin color segmentation. This approach can be subdivided into parametric approach (used by Lee and Yoo [10], and non- parametric approaches (used by Jones and Rehg. [1]). Parametric model approaches represent the skin color distribution in parametric form, such as single Gaussian method and mixture of Gaussian method, described by Vezhnevets et al. [11], while elliptical boundary model is described and used by Lee and Yoo. [10].

Parametric skin modeling methods are better suited for constructing classifiers in case of limited training and expected target data set. The generalization and interpolation ability of these methods makes it possible to construct a classification with acceptable performance from incomplete training data [11]. On the other hand, Jones and Rehg [1] use the non- parametric approach. The key of this approach is to estimate color distribution from the training data without deriving an explicit model of the skin color. The histograms are used to represent density in color space.

\subsection{Bayes Classifier}

The value of Pskin (RGB) computed in equation (1) is actually a conditional probability $P(R G B \mid$ skin $)$. This is a probability of observing a pixel of color $(R G B)$, knowing that a skin pixel is seen. A more appropriate measure of skin detection would be $P(\operatorname{skin} \mid R G B)$ which is computed by Bayes rule [1][11][3]: 


$$
\operatorname{Pskin}(R G B)=\frac{\operatorname{skin}[R G B]}{\operatorname{Norm}}
$$

$$
P(\operatorname{skin} \mid R G B)=\frac{P(R G B \mid \text { skin }) \cdot P(\text { skin })}{P(R G B \mid \text { skin }) \cdot P(\text { skin })+P(R G B \mid \sim \text { skin }) \cdot P(\sim \text { skin })}
$$

$P(R G B \mid$ skin $)$ and $P(R G B \mid \sim$ skin $)$ are directly computed from skin and nonskin histograms. The prior probabilities $P($ skin $)$ and $P(\sim$ skin $)$ can easily be estimated from the overall number of skin and non- skin pixels in the training set as is shown below [1][3]:

$$
\begin{aligned}
& P(\text { skin })=\frac{T_{s}}{T_{s}+T_{n}} \\
& P(\sim \text { skin })=\frac{T_{n}}{T_{s}+T_{n}}
\end{aligned}
$$

Where, $\left(T_{s}\right.$ and $\left.T_{n}\right)$ are the numbers of skin and non- skin pixels respectively.

Tretyakov [12] states that if the values of $P(w)$ and $P(R G B \mid w)$ are known for $(W=\{$ skin, nskin $\}), P(w \mid R G B)$ will be determined, which is already a nice achievement that allows to use the following classification rule:

If $P(\operatorname{skin} \mid R G B)>P(\sim \operatorname{skin} \mid R G B)$ then $(R G B)$ is classified as skin pixel, otherwise its classified as non-skin pixel. This is the so-called maximum a posteriori probability rule. Using the Bayesians' formula, the classification rule can be transformed to the:

If $P(R G B \mid$ skin $) / P(R G B \mid \sim$ skin $)>p(\sim$ skin $) / p($ skin $)$ then $(R G B)$ is classified as skin pixel, otherwise it is non-skin pixel.

Chai, Bouzerdoum [13] and Tretyakov [12] define Bayes decision rule for minimum cost (loss, risk). This rule can be used to classify $(R G B)$ into skin color class $\left(w_{1}\right)$ or non-skin color class $\left(w_{2}\right)$. Let $\left(C_{i j}\right)$ denotes the cost of deciding $\left(R G B C w_{i}\right)$ when $\left(R G B \in w_{j}\right)$. $\left(C_{i j}\right)$ here represents the cost of correct classification when $(i=j)$, and false classification when $(i \neq j)$.

The Bayesian decision rule formula for minimum cost (Bayes' classifier) is [13][3]:

$$
\begin{aligned}
& \frac{P(R G B \mid \text { skin })}{P(R G B \mid \sim \text { skin })}>\theta \rightarrow R G B \in \text { skin } \quad\left(w_{1}\right) \\
& \frac{P(R G B \mid \text { skin })}{P(R G B \mid \sim \text { skin })}<\theta \rightarrow R G B \in \sim \text { skin }\left(w_{2}\right)
\end{aligned}
$$

Where, 
$\theta=\frac{C_{12}-C_{22}}{C_{21}-C_{11}} \cdot \frac{P(\sim \text { skin })}{P(\text { skin })}$

$(\theta)$ represents the adjustable threshold, where the costs of false classifications are manipulated by $\left(C_{12}\right.$ and $\left.C_{21}\right)$ for false detection and false dismissal, respectively, while the costs of correct classifications (i.e. $C_{11}$ and $C_{22}$ ) are typically set to zero. This means that the final $(\theta)$ value is calculated by the following equation:

$\theta=\frac{C_{12}}{C_{21}} \cdot \frac{P(\sim \text { skin })}{P(\text { skin })}$

\section{Pornographic Images Detector}

When dealing with detection of nudity, it is important to have a good method of recognizing skin regions [14]. The first step in pornographic images detector is skin detection. The output of this detector is then used to extract features vector [2]. The final task is then to find the decision rule on this features vector that optimally detects pornographic images from those not [4]. The features vector is computed for each image in the training set. Then it is used to train a classifier on these features to determine whether a nudity or pornography for any given image is present or not [1]. In order to learn a classifier a set of lablled images are given $D=\left\{\left(x_{i}, t_{i}\right)\right\}, x_{i} \in X, t_{i} \in\{0,+1\}$, where, (xi) represents the features vector for $\left(\right.$ image $\left._{i}\right),(X)$ stands for the features vector for all images in the training set, and $\left(t_{i}\right)$ indicates whether (image $\left.{ }_{i}\right)$ is considered porn $\left(t_{i}=1\right)$ or non-pornographic $\left(t_{i}=0\right)$ [15].

The naïve Bayesian classifier [16][17][18] is adopted and used as the decision rule to decide whether an image is pornographic or not. It is written in context of pornographic images detector as shown in the following equation:

$$
P(C=c \mid \vec{X}=\vec{x})=\frac{P(C=c) \cdot \prod_{i=1}^{|v|}\left(P\left(X_{i}=x_{i} \mid C=c\right)\right.}{\sum_{k \in\{\text { porn }, \sim \text { porn }\}} P(C=k) \cdot \prod_{i=1}^{|v|} P\left(X_{i}=x_{i} \mid C=k\right)}
$$

Where, $C=\{$ Porn, $\sim$ porn $\}$, and $\vec{X}$ Represents the features vector. $P\left(X_{i}=x_{i} \mid C=c\right)$ and $P(C=c)$ can easily estimated as relative frequencies from the training set. Equation 10 estimates $P\left(X_{i}=x_{i} \mid C=\right.$ pon $)$ and equation 11 estimates $\mathrm{P}(\mathrm{C}=$ Porn $)$.

$P\left(X_{i} \mid\right.$ Porn $)=\frac{N P x}{N P}$

Where, $(N P x)$ represents Number of pornographic images in pornographic images detector training set that have the feature $(x)$ 
satisfies a predefined threshold, and $(N P)$ represents the total number of pornographic images in pornographic images detector training set. The probability of porn image is:

$$
P(C=\text { porn })=\frac{N P}{N T}
$$

Where, $(N P)$ is the total number of pornographic images in pornographic images detector training set, and (NT) represents the total number of all images in the training set (pornographic and non-pornographic images).

The flowchart in figure (2) illustrates the training phase of the proposed pornographic images detector which uses naive Bayesian classifier. It is trained on the features of pornographic and nonpornographic images.

The following decision rule is used to decide whether an image is pornographic or not.

$$
\frac{P(C=\operatorname{Porn} \mid \vec{X}=\vec{x})}{P(C=\sim \operatorname{porn} \mid \vec{X}=\vec{x})}>\lambda
$$

Where $(\lambda)$ is the threshold to distinguish between pornographic and nonpornographic images, which is determined empirically. Figure (1) illustrates the classification process of an image to show whether it is pornographic or not.

\subsection{Features Extraction Process}

Nude images can be detected by using combination of simple visual clues-color, texture, and elongated shapes - and class -specific grouping rules [19]. The features vector is composed of information about the color and size of skin regions. If no skin regions can be found, then the image is classified as non- pornographic. Among these features, composing a features vector, are: the average skin probability of the whole image [2], number of skin regions in the image [2][4][3], distance from the centroid of the largest skin region to the center of the image [5], ratio of skin area to image area [8], ratio of the area of the largest skin segment to the image [20][3], height and width of image, size in pixels of the largest connected component of skin [1], and mean and standard deviation of the skin region [6]. The features can be broadly grouped into ones making use of skin color information, and others which don't.

\subsubsection{Skin Independent Features}

The size of an image acts as a fast and basic filter. If an image dimensions are below a predetermined threshold, for example $100 \times 100$ pixels, the image is automatically labeled as non-porn image. This image is probably a thumb. Most of the features for images that are smaller than 100 x 100 pixels can't be computed. These images are unlikely to contain 
recognizable pornographic-content in any case. Similarly, an image that contains only a limited number of colors (for example, less than one hundred) is labeled as non-pornographic image. This is because it is unlikely to be photographic image [8][3].

\subsubsection{Skin Dependent Features}

Based on the strong correlation between pornographic images and large skin patches, features depending on skin are extracted after detecting skin pixels in the image [2]. The features vector is computed and used to train the proposed pornographic images detector. The skinbased features are more important, since pornography contains significant portion of skin pixels [5]. Several features depend on skin or not are described below[3]:

- The Image Dimensions

- Percentage of Pixels Detected as Ski

- Number of Connected Components of Skin Pixel

- Number of Connected Components of Holes Pixels

- Average Probabilities of Skin Pixels

- The Size in Pixels of Largest Connected Components of Skin

- Features Based on Informal Observation.

\section{Practical Part}

\subsection{Pornographic Images Detection System Structure}

The structure of the proposed pornographic images detection system is shown in figure (1). The first step is Size and Colors Analysis Filter. The second step is Skin detection and segmentation. The third step is the extraction of the features from the skin region obtained by the second step. The fourth step represents the naive Bayesian classifier takes two inputs; one is the features vector and the other is the probability values of training porn and non-porn images. The output on the other hand, is a real number $(D)$, which can be compared to a threshold $(\lambda)$. This comparison will decide whether the input color image is a pornographic image or not. The value of $(\lambda)$ is determined empirically. 


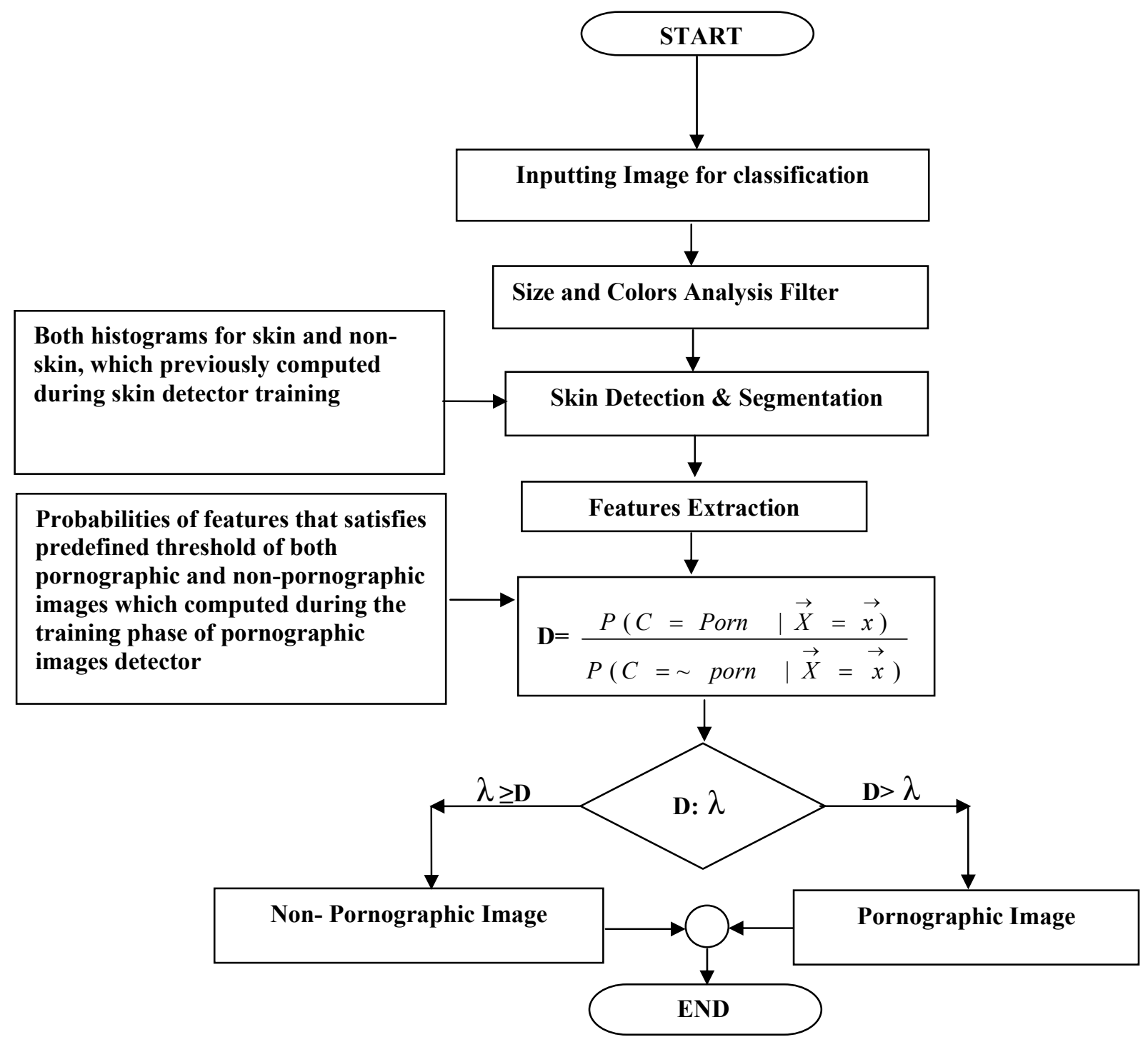

Figure (1) The proposed pornographic image detector based on Naive Bayesian classifier

One hundred and thirty six (136) images are used to train the pornographic images detector. These images are manually classified as pornographic and non-pornographic image sets. The pornographic set includes eighty nine (89) images whereas the non-pornographic set includes forty seven (47) images. The features of both sets are extracted and computed. These feature vectors are used to train the naive Bayesian classifier. Figure (2) illustrates the training phase of the pornographic images detector. 


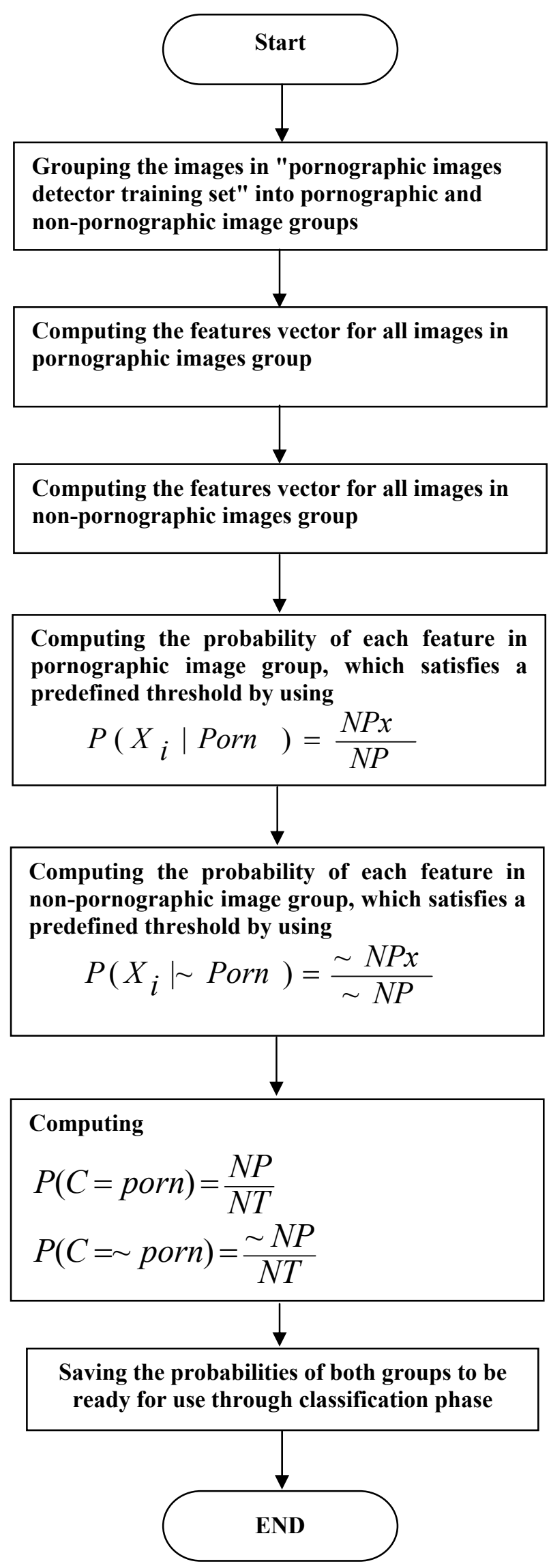

Figure (2): The training phase of naive Bayesian classifier used in proposed pornographic images detector 


\subsection{Skin and non-Skin Histograms}

The histogram of an image represents the relative frequency of the various colors occurrence in that image [21]. Umbaugh [22] considers it as the distribution of the colors in an image. The skin and non- skin histograms of this work are constructed by using RGB color model. Each channel has 256 bins. This means that the histogram has 16777216 colors In order to reduce computation time, and memory spaces, a $256 \times 256 \times$ 256 colors is quantized into a $128 \times 128 \times 128=2097152$ colors in RGB color space. Each channel of both histograms (skin and non- skin) is divided into (128) bins. Each bin in a histogram stores an integer which represents the number of times that color value occurred in the skin classifier training set. The training data set contained (251) photos are used to build skin and non-skin histograms. The images in this set have been manually separated into ones containing skin (119 images) and others not containing skin (132 images). Skin pixels have been manually labelled in the set of skin images.

\subsection{Bayesian Decision Rule Based Skin Detector}

The state -of- art relating to skin - tone detection in images is based on probability distribution in color spaces. Typically to decide whether a pixel belongs to a skin - region is made on the basis of only pixel-color value. The skin and non- skin histogram models are used in training phase of skin pixel classifier. Given a skin and non-skin histograms, the probability of each color value in both histograms could be computed by using equations 13 , and 14 respectively.

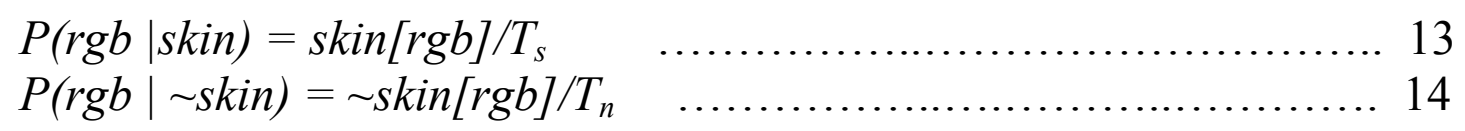

Where, skin [rgb] is the pixel count contained in bin rgb of the skin histogram, $\sim$ skin $[\mathrm{rgb}]$ is the equivalent count in the non-skin histogram, and $\left(T_{s}\right.$ and $\left.T_{n}\right)$ are the total counts contained in the skin and non-skin histograms, respectively.

All the probabilities obtained from equation 13, and 14 are kept and used during a new skin pixel detection. Figure (3) illustrate the training phase of skin pixel classifier. While Figure (4) illustrates the skin detection based on Bayes classifier and segmentation process. $(\theta)$ represents threshold value which determined empirically. 


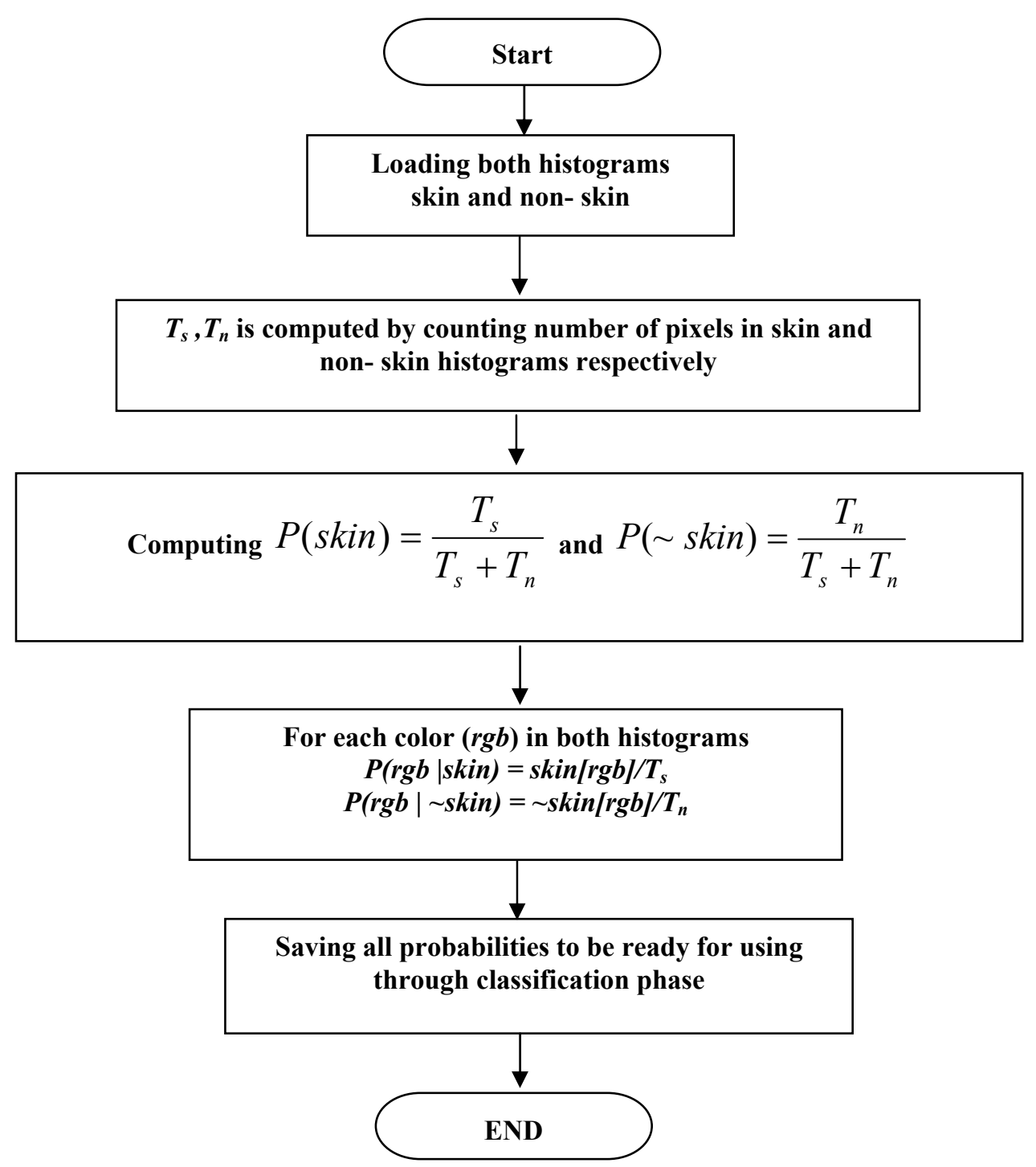

Figure (3): Training phase of skin pixel classifier 


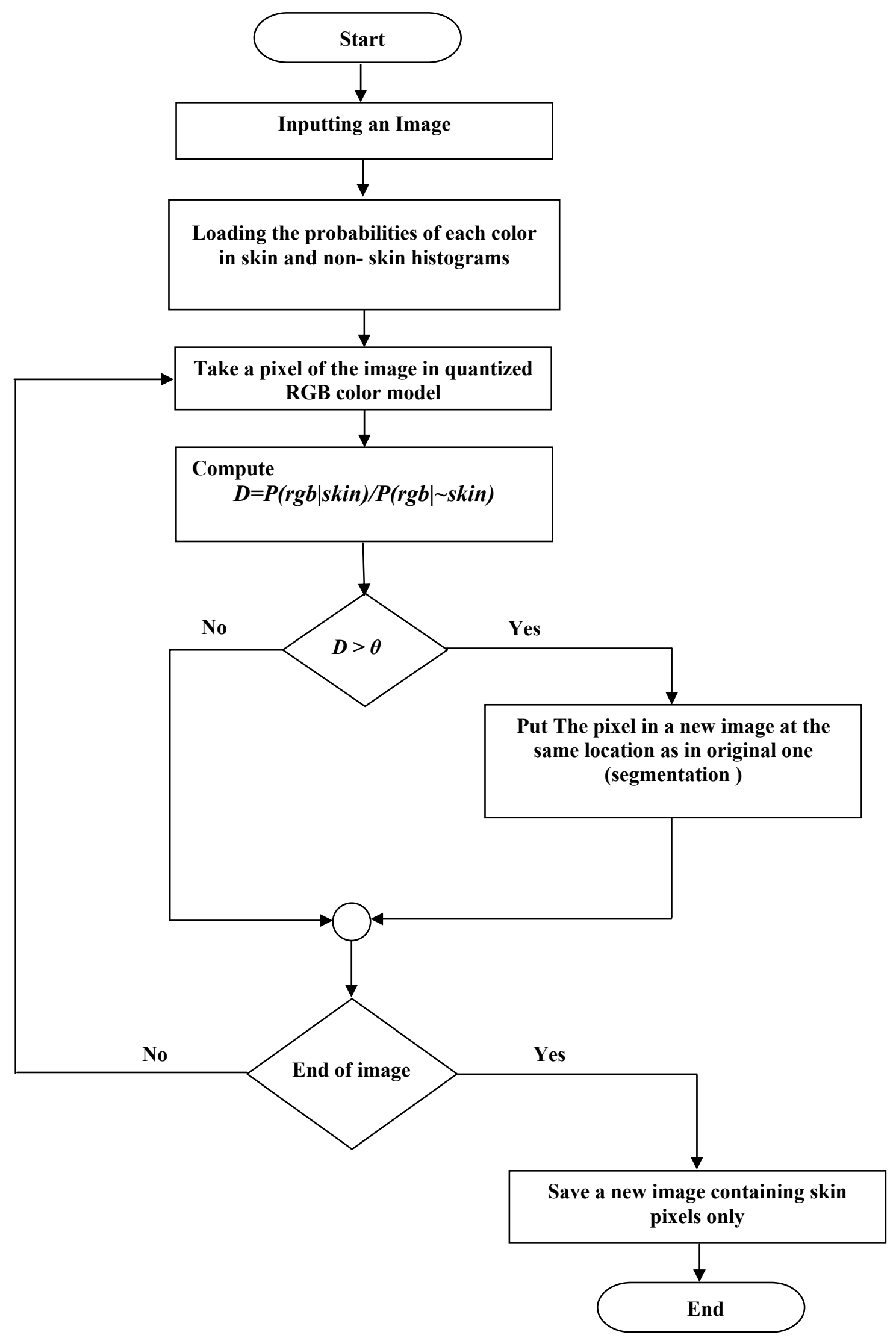

Figure (4): Skin detection and segmentation processes 


\subsection{Evaluation Metrics}

A skin detection process is never perfect and different users use different criteria for evaluation its performance. General appearance of the size zones detected is one of the evaluation criteria. In order to quantify the performance evaluation, True Positive (TP), False Positive (FP), True Negative (TN) and False Negative (FN) are computed for all pixels in "Skin classifier testing set" through testing skin detector. (FP) is the proportion of non-skin pixels classified incorrectly as skin. On the other hand, (TP) is the proportion of skin pixels classified correctly as skin. (TN and FN) are the complements of (FP and TP) respectively. Four metrics are used to evaluate the performance of skin detector. [23][9][3]:

Recall $=T P /(T P+F N) \quad$............................... 15

Precision $=T P /(T P+F P) \quad$.............................. 16

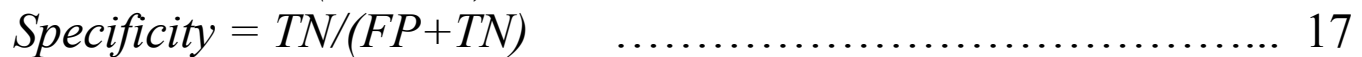

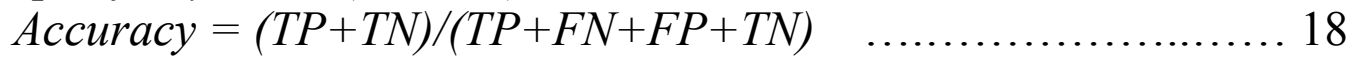

The performance of skin detector can be quantified by computing the ROC (Receiver Operating Characteristics) curve [23] which measures the threshold-dependent trade-off between false positive rate and true positive rate.

\section{Results of Pornographic Image detector}

The experimental results are presented to show the effectiveness of the proposed pornographic images detector which is based on naïve Bayesian classifierl. All the experimental results obtained throughout the constructing, implementing, and testing of the pornography images detection, are described and analyzed in this section.

\subsection{Skin Detector Testing}

The implementation of skin detector is tested in different images with simple and complex backgrounds, indoor and outdoor, image size and skin colors. The experiment is performed on "Skin classifier testing set", which includes (260) uncontrolled (different illumination, captured quality, distance to camera, etc.) images. Each of the first (171) images contains an arbitrary number of pornography images and images containing an arbitrary number of people and faces. The other (89) images contain no people (no skin pixels). Images with objects that present skin-like tones (such as red flower, a dog, chocolate, etc.) are included too.

The testing classifier set has been used to demonstrate the skin detector performance at various threshold values. The effect of threshold values on detection results is demonstrated in figure (5). The value of threshold is determined empirically so as to reduce the amount of false detection. The original image is shown in figure $(5 \mathrm{~g})$. The images shown 
in figures ( $5 \mathrm{a}, \mathrm{b}, \mathrm{c}, \mathrm{d}, \mathrm{e}$, and $\mathrm{f}$ ) represent the output of Bayesian decision rule-based skin detector by using different threshold values equaling to $(0.02,0.2,0.4,0.6,0.7$, and 0.8$)$ respectively.

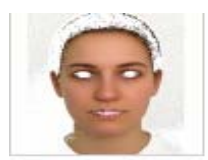

(a)

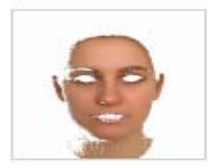

(d)

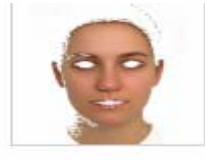

(b)

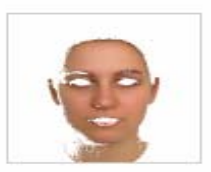

(e)

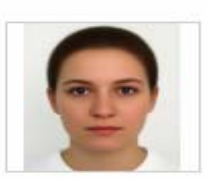

(9)

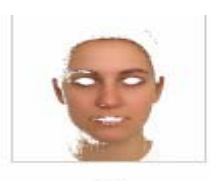

(C)

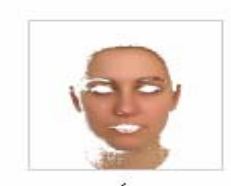

(f)

Figure (5): Sample results of Bayes decision rule-based skin detector by using different threshold values

The same conditional probability of skin and non-skin colors and the same threshold value equaling (0.6) are used for all tested images. The detection does good job of detecting skin in most of these examples. The images produced by Bayesian decision rule based-skin detector in comparison with their originals show clean skin pixels even when light conditions vary, as presented in figure (6).
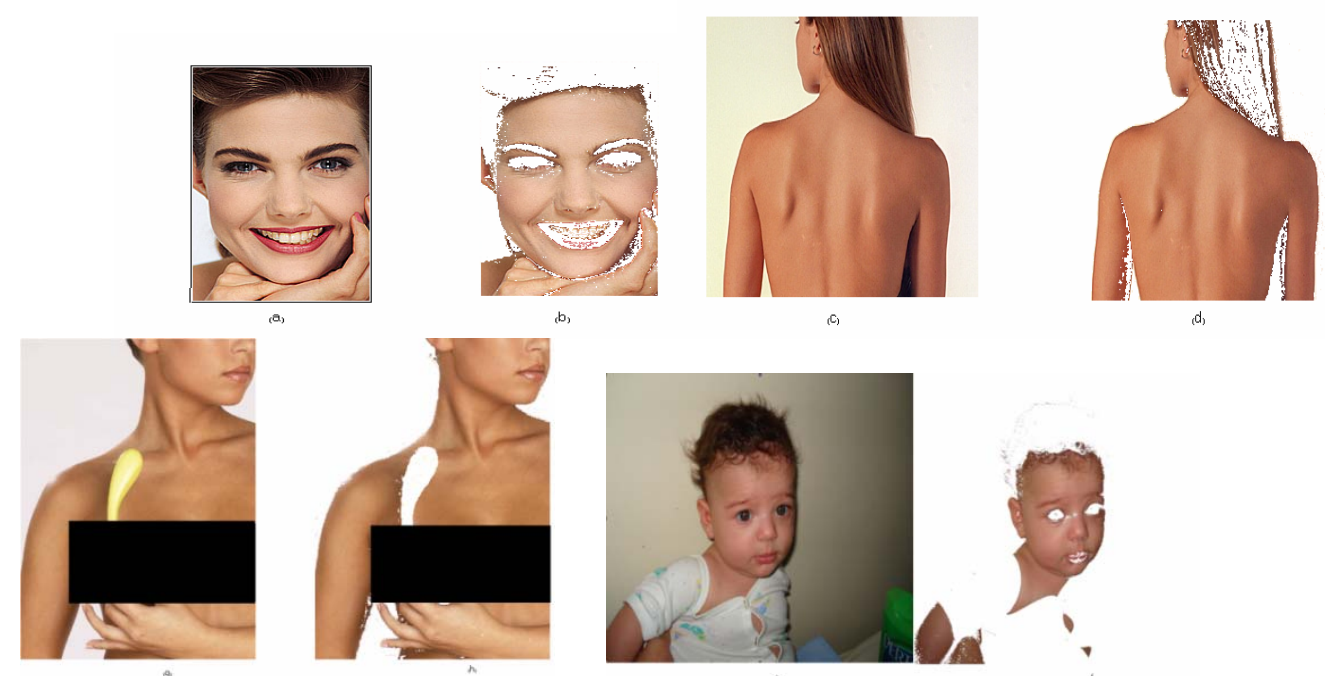

Figure (6): Examples of the results obtained by using Bayes decision rule based skin detector

The detector tends to fail on highly shadowed or over exposure skin. Figure (7 b) shows example of the former type of failure, whereas figure $(7 \mathrm{~d})$ shows that latter one. 

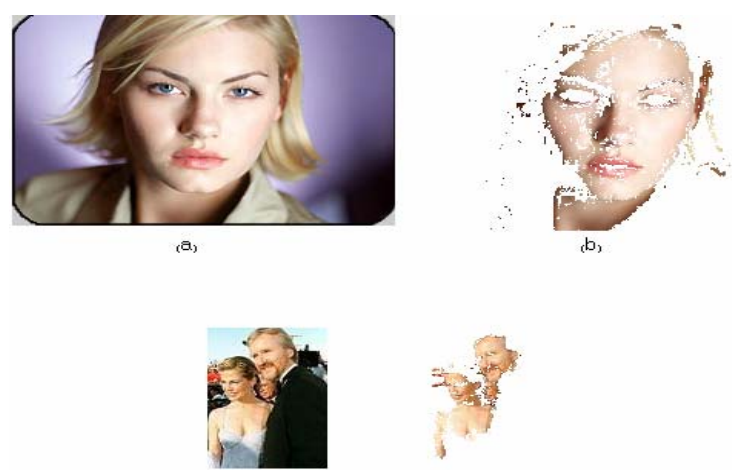

(c)

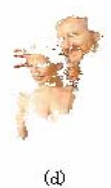

Figure (7): Two examples of the former type of failures in detecting skin pixels, which obtained by using Bayes decision rule based skin detector

Figure (8) also shows very complex images with different size groups of skin. Couple of which (figure $8 \mathrm{~b}$ and $\mathrm{d}$ ) versus their originals (figure $8 \mathrm{a}, \mathrm{c}$ ) not correctly classified as they are very similar to skin in color, whereas the skin pixels within image in figure $(8 \mathrm{e})$ is detected correctly except little number of scattered pixels incorrectly detected as skin.

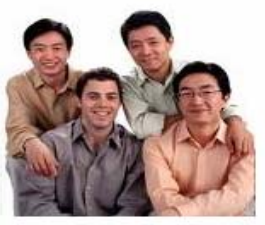

(a)

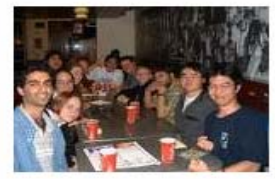

(C)

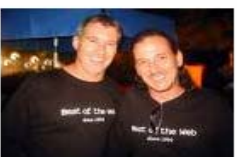

(e)

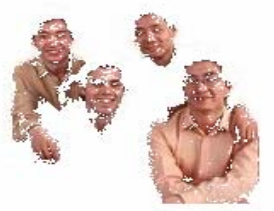

(b)

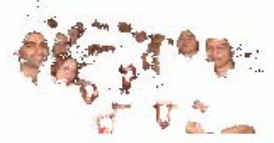

(d)

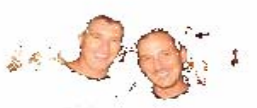

()

Figure (8): Several complex examples and the results obtained by using Bayes decision rule based skin detector

The performance of the detector on non-skin pixels is shown in figure (9). The false detection in images (figures (9 b), and (9 d) are sparse and scattered while no false detection pixels are obtained in images. 9 e and f). 

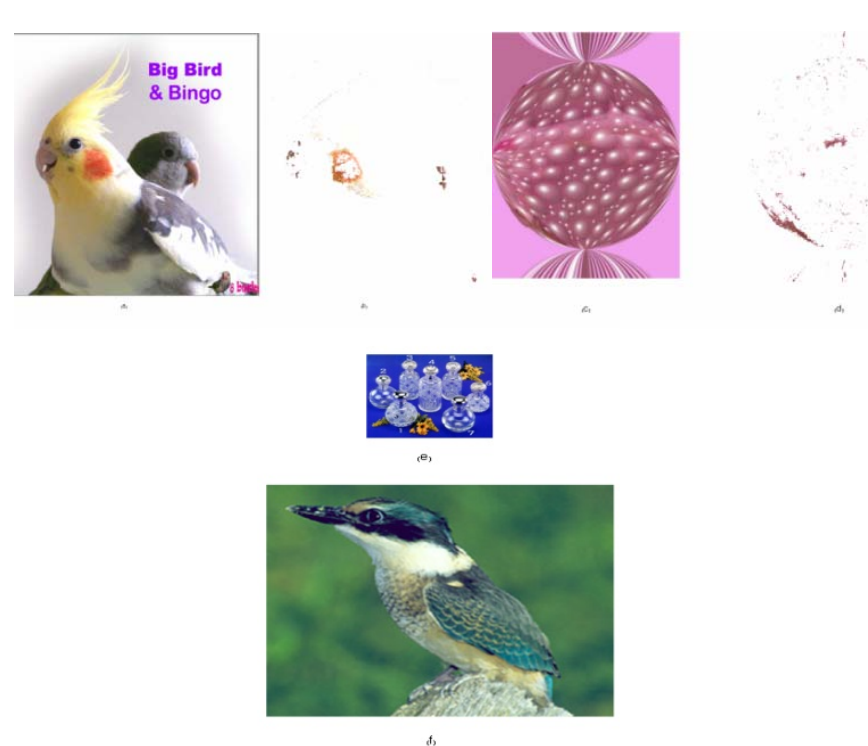

Figure (9): More different examples and the results obtained by using Bayes decision rule based skin detector

More problematic are images with wood, copper colored metal or chocolate (figure.10) as they contain colors often occurring in the skin model and are difficult to reliably discriminate. This result is fairly dense sets of false positives.
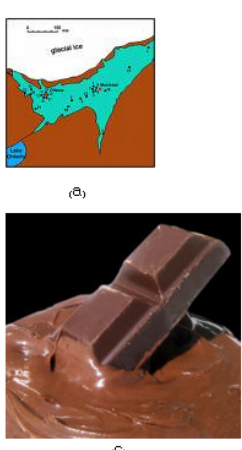
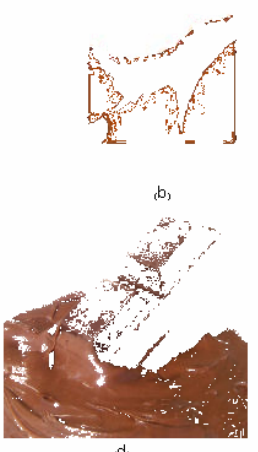

Figure (10): Non-skin pixel examples detected wrongly as skin pixels by using Bayes decision rule based skin detector

\subsubsection{Skin Detector Evaluation}

In order to evaluate the performance of Bayes decision-based skin detector, the number of skin pixels detected correctly must be known (TP). The evaluation metrics are used for six different threshold values $(0.02,0.2,0.4,0.6,0.7$, and 0.8$)$. The figure (11) illustrates the evaluation values (sensitivity, precision, specificity, accuracy and error) based on the number of pixels. 


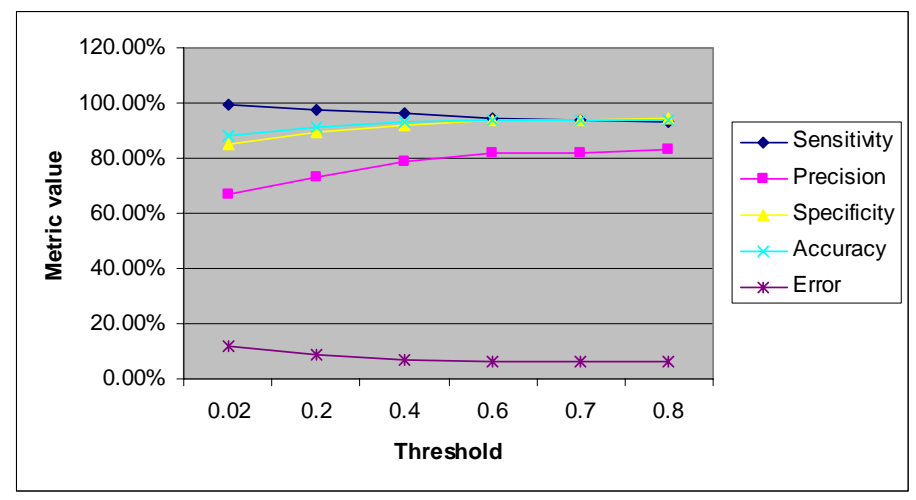

Figure (6.11): The evaluation values based on the number of pixels.

The performance of Bayesian decision rule-based skin detector at different threshold values are summarized in the ROC as shown in figure (6.12). The axis labeled "Rate of true positive" gives the fraction of pixels classified correctly as skin (sensitivity), while "Rate of false positive" gives the fraction of non-skin pixels classified incorrectly as skin (1specificity).

Considering the unconstrained nature of Internet images, the performance of the skin detector is surprisingly good. The best performance can detect $(94.29 \%)$ of skin pixels with a false positive rate $(6.48 \%)$, by using Bayesian decision rule -based skin detector. These rates correspond to the marked point on the ROC curve where the Euclidean distance to the optimal performance point $(0,1)$ is the minimum distance. The threshold value that satisfies the best performance is 0.6 .

Another scalar measure of detector performance is the area under ROC curve. An area of (1) represents a perfect detector, while an area of (0.5) represents a worthless detector. The closer the curve follows the left-hand border and then the top border of the ROC space, the more accurate the detector is. The true positive rate is high and the false positive rate is low. Statistically, more area under the curve means that it is identifying more true positives while minimizing the false positive rate as shown in figure (6.12).

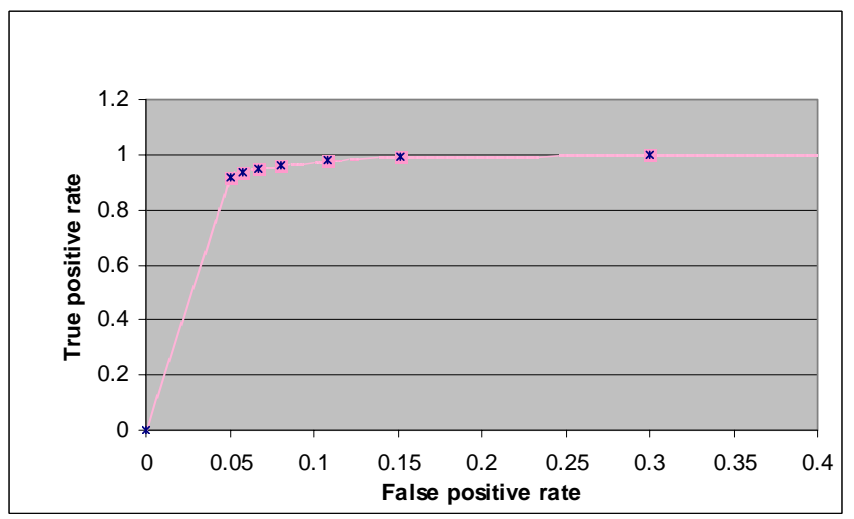

Figure (6.12): ROC curve of Bayes decision rule-based skin detector 
Although there is no ability to locate any two papers which used the same test sets, it may be useful to examine the reported results which have been published. The performance of the Bayes decision rule basedskin detector in this thesis is compared with the performance of other skin detectors. The TFOM (Bethe Tree Approximation of First Order Model) model proposed by Zheng et al. [2] can detect (72\%) of skin pixels with $(5 \%)$ false positive rate. Whereas, the proposed Bayesian model by Jones et al. [1] can detect $(69 \%)$ at the same false positives. It also can detect $(80 \%)$ of skin pixels with $(8.5 \%)$ false positive rate, or $(90 \%)$ correct detection with (14.2\%) false positives. The recall rate of pixel based skin color classification proposed by Gasparini et al. [9] is (92\%) while the precision equals (39\%). The best performance of the Bayes decision rule based-skin detector can detect $(94.29 \%)$ of skin pixels correctly with false positive equals to $(6.48 \%)$. The values of precision, specificity and accuracy equal to $(81.58 \%, 93.52 \%, 93.7 \%)$ respectively. These values indicate that the Bayes decision rule based-skin detector outperforms all the skin detectors mentioned above.

\subsection{The Pornographic Images Detector Testing}

Testing experiments of pornographic images detector are described in this section. "Pornographic images detector testing set" contains (154) images which are used to test the pornographic images detector performance. The testing set is divided into two groups; the first includes (94) pornographic images only and the second (60) non-pornographic images. In the testing set, a features vector based on the output of skin detector is computed for each image. The decision rule in equation 12 is used on features vector that optimally separates pornographic images from non-pornographic. To determine the best threshold value several experiments have been done on the testing set, using different threshold values. The best results are obtained when the threshold value $(\lambda)$ equals (0.4). The first row of figure (13) shows several non-pornographic images correctly classified, while the second row of the same figure shows some of the pornographic images correctly classified.
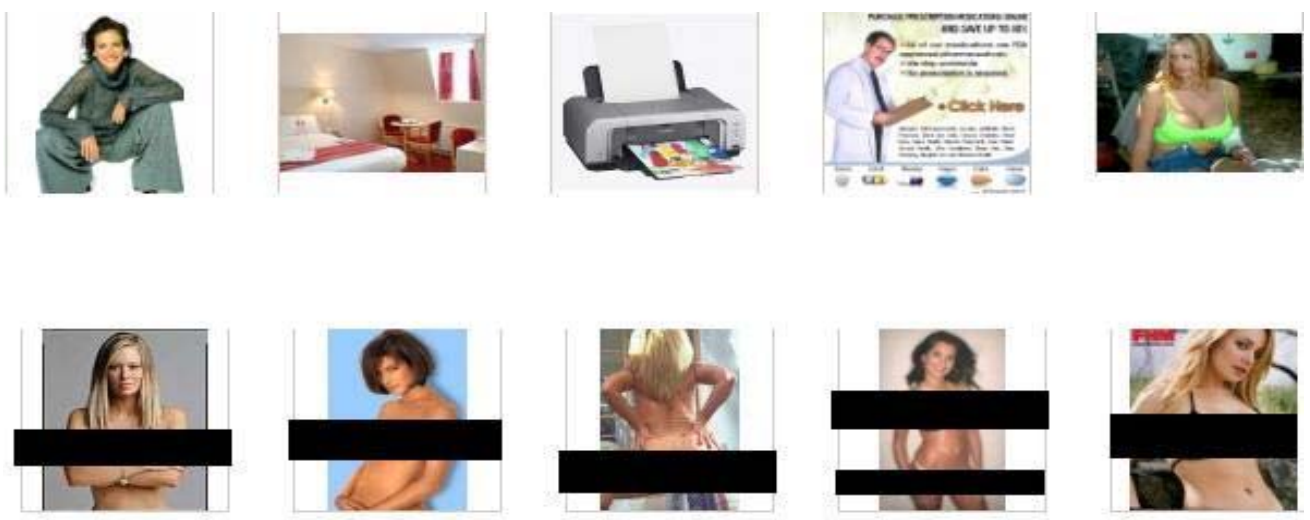

Figure (13): Examples of the results obtained by pornographic images detector 
The pornographic images in figure (14 a) are not correctly detected as far as the skin appears almost white due to over exposure, and thereby most of the skin is not detected. Since some areas of the body are covered with cloth, the skin rate of the tested image is to be minimized. The pornographic image in figure (14 b), on the other hand, is not correctly classified since it has more than one skin region area. The horizontal -or the vertical- window includes neither high rate of skin pixels nor high rate of skin connection pixels. Besides, the maximum skin region of this image does not satisfy that of pornographic images.

(a)

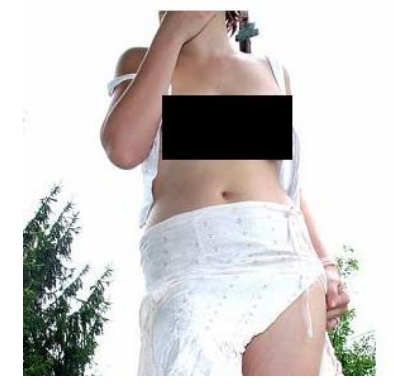

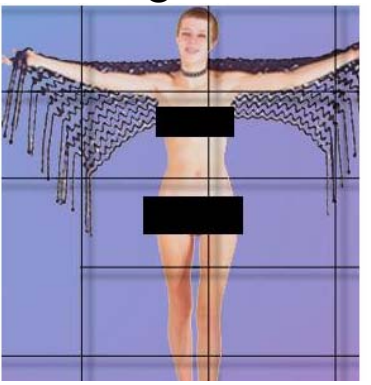

(b)

Figure (14): Two examples of the former type of failures in detecting pornographic images by using pornographic images detector

The image in figure (15 a) is benign, yet is incorrectly detected as pornographic since the cloth take a skin- like color. Whereas, the image in figure (15 b) which is a portrait is incorrectly detected as pornographic due to the many skin pixels it exposes.

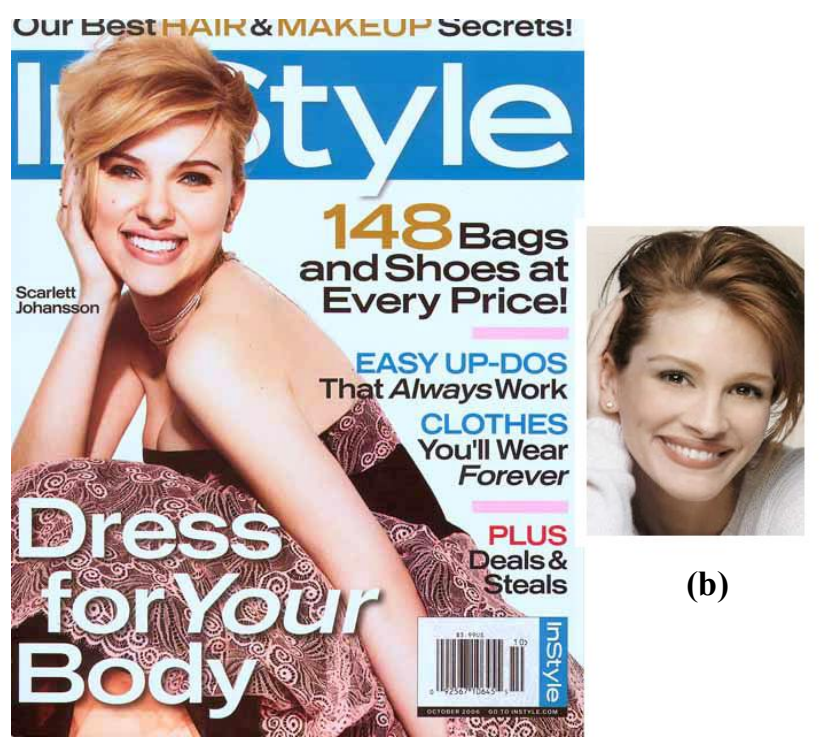

(a)

Figure (15): Non-pornographic images incorrectly detected as pornographic images

The dark pornographic images are not correctly detected. The dark skin regions are as similar as the shadow skin region that skin detector could not correctly detect as skin. Figure (16) shows the dark skin images incorrectly classified as non-pornographic. 


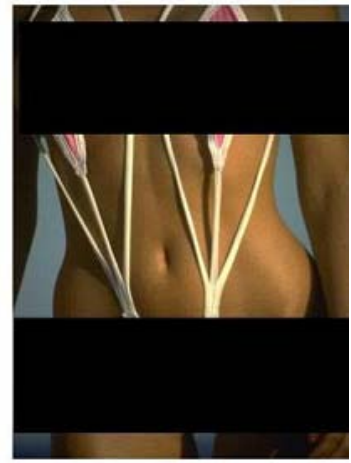

Figure (16): Dark skin pixels pornographic image incorrectly detected as non-pornographic image

\subsection{The Pornographic Images Detector Evaluation}

The pornographic images detector performance is evaluated by using the same approach as in the evaluation of skin detector. In order to quantify the performance evaluation of pornographic images detector, TP, FP, TN and FN are computed on all images in "Pornographic images detector testing set" through testing the pornographic images detector. (FP) represents the number of non pornographic images incorrectly classified as pornographic. On the other hand, (TP) represents the number of pornographic images correctly classified as pornographic images. (TN and $\mathrm{FN}$ ) are the complements of (FP and TP) respectively. The recall, precision, specificity, and accuracy metrics are used to evaluate the performance of pornographic images detector as described in section 4.4. Eighty six (86) out of ninety four (94) images (91.48\%) containing pornography are correctly detected. And (56) out of (60) images $(93.33 \%)$ containing no pornography are correctly detected. The FP rate is $(6.67 \%)$. The statistical summary of the evaluation pornographic images detector performance is shown in table (1). Evaluation metrics (sensitivity, precision, specificity and accuracy) are summarized in table (2).

Table (1): Statistics summary of evaluating pornographic images detector

\begin{tabular}{|l|c|c|c|c|}
\hline \multicolumn{1}{|c|}{ Image sets } & $\begin{array}{c}\text { Number of } \\
\text { images } \\
\text { correctly } \\
\text { detected }\end{array}$ & $\begin{array}{c}\text { Number of } \\
\text { images } \\
\text { incorrectly } \\
\text { detected }\end{array}$ & $\begin{array}{c}\text { Rate of } \\
\text { images } \\
\text { correctly } \\
\text { detected }\end{array}$ & $\begin{array}{c}\text { Rate of } \\
\text { images } \\
\text { incorrectly } \\
\text { detected }\end{array}$ \\
\hline $\begin{array}{l}\text { Pornographic } \\
\text { images }\end{array}$ & 86 & 8 & $91.48 \%$ & $8.52 \%$ \\
\hline $\begin{array}{l}\text { Non-Pornographic } \\
\text { images }\end{array}$ & 56 & 4 & $93.33 \%$ & $6.67 \%$ \\
\hline
\end{tabular}

Table (2): Statistics summary of evaluating pornographic images detector

\begin{tabular}{|c|c|}
\hline Metric & Metric value \\
\hline Sensitivity (Recall) & $91.48 \%$ \\
\hline Precision & $95.55 \%$ \\
\hline Specificity & $93.33 \%$ \\
\hline Accuracy & $92.20 \%$ \\
\hline Error & $7.80 \%$ \\
\hline
\end{tabular}


High sensitivity means that the detector is highly sensitive as to pornographic features. Whereas, precision equaling (95.55\%) indicates that several non- pornographic images are detected as pornographic. The behavior of the pornographic images detector is to detect high rate of pornographic images in addition to a few rate of false positive. The specificity value is confirmed numerically that the rate of false detecting is low. This means that the pornographic images detector detects $(6.67 \%)$ of non-pornographic images incorrectly as pornographic images. Finally the error rate is $(7.8 \%)$. This means that the detector detects $(7.8 \%)$ of all images incorrectly as pornographic images or non-pornographic images. Since there is no Naive Bayesian based pornographic images detector published, the performance evaluation of the proposed pornographic images detector is compared to that of the systems published. The pornography detector proposed by Lin et al. [5] has the total accuracy of (74.61\%), false positive rate equals $(34.98 \%)$ and false negative rate of (13.98\%).

The FP and FN rates could be estimated from the ROC curve for the adult image detector performance proposed by Zheng et al. [4]. These rates equal (9\% and 20\%) respectively. Finally, Liang and et al. [8] proposed a system for the detection pornographic images by using multiple features derived from skin regions with MLP neural network classifier. The error rates obtained from two different experiments equal $(18 \%$ and $11 \%)$ respectively. The sensitivity (recall), precision, specificity, accuracy, error and false positive rate of the proposed pornographic images detector equal to $(91.48 \%, 95.55 \%, 93.33 \%, 92.2 \%$, $7.8 \%, 6.67 \%$ ) respectively. Comparison between the performance of the proposed pornographic images detector and those published indicates that the proposed detector outperforms all the above- mentioned pornographic images detectors.

\section{Conclusion and Future Work}

\subsection{Conclusions}

The pornographic image detector based on the naive Bayesian classifier represents the visual anti-spam filter. This filter is evaluated over a data set consisting of (94) pornographic images and (60) nonpornographic images by using sensitivity, precision, specificity and accuracy metrics. The performance evaluation of the pornographic images detector shows a detective rate of $(91.48 \%)$, high total accuracy of $(92.20 \%)$, true positive of $(91.48 \%)$, false -alarm rate of $(6.67 \%)$, and miss detection of $(8.52 \%)$ when the threshold value $(\lambda)$ equals $(0.4)$. The following conclusions are obtained throughout both testing and evaluating of pornographic images detector: 
1. The accuracy of the Bayesian decision rule-based skin detector equals to $(93.7 \%)$. It could be achieved with a false positive rate of $(6.48 \%)$ and true positive rate of $(94.29 \%)$ when the threshold value $(\lambda)$ equals $(0.6)$.

2. Mistakes by skin detector occur for several reasons. In some test images, most or all of the skin area is so dark, shadowed, or over exposure, which makes the skin detector fail with false negative rate equals to $(5.71 \%)$.

3. The sensitivity (recall), precision, specificity, accuracy and error and false positive rate of the proposed pornographic images detector equal to $(91.48 \%, 95.55 \%, 93.33 \%, 92.2 \%, 7.8 \%$, and $6.67 \%)$ respectively when the threshold value $(\lambda)$ equals $(0.4)$. These results suggest that color can be a more powerful cue for detecting pornographic images in unconstrained images as those found within e-mail messages.

4. Although the error rate is $(7.8 \%)$ for the pornographic images detector, it sound particularly good. It must be emphasized that the non-porn images in the data set are non-trivial, in other words most of images contain large skin -like pixels, the nudes are very small, or the skin area is dark.

\subsection{Future Work}

This research suggests several interesting points for future work as follows:

1. The current implementation of pornographic images detector uses only the skin region features and simple informal observation features. The performance could be improved substantially by techniques such as adding a face detector. This detector would be useful in avoiding some of false positives; for example, full-face portraits are classified incorrectly as pornographic images since they are including many skin pixels.

2. The skin pixels detector of this work is based on Bayesian decision rule. The obtained results are promising, but more promising results require further studies on other methods like detecting skin regions based on skin texture. 


\section{Reference}

1) Jones, M. J. and Rehg, J. M., 2000, "Statistical Color Models with Application to Skin Detecting", International Journal of Computer Vision ( IJCV), Vol.1, No. 46, pp. 81-86.

2) Zheng, H., Liu, H., and Daoudi, M., 2004, "Blocking Objectionable Images: Adult Images and Harmful Symbols", Proceedings of IEEE International Conference on Multimedia and Expo, Taipei, Taiwan, 27-30 June. January 28-30.

3) Taqa, Alaa Yaseen, 2007, "Constructing anti spam filter based on naïve Bayesian classifier", Ph.D. thesis, computer science Dep., Computers sciences and mathematics college, Mosul University.

4) Zheng, H., Daoudi, M., and Jedynak, B., 2004, "Blocking Adult Images on Statistical Skin Detection", Electronic letters on Computer Vision and Image Analysis, Vol. 4, No. 2, pp. 1-14.

5) Lin, Y. C., Tseng, H. W., and Fuh, C. S., 2003, "Pornography Detection Using Support Vector Machine", 16th IPPR Conference on Computer Vision, Graphics and Image Processing (CVGIP), Kinmen, 17-19 August.

6) Rowley, H. A., Jing, Y., and Baluja, S., 2006, "Large Scale ImageBased Adult-Content Filtering", 1st International Conference on Computer Vision Theory and Applications (VISAPP), Setubal, Potugal, 25-28 February.

7) Albiol, A., Torrest, L. and Delp, E. J., 2001, "Optimum Color Spaces For Skin Detection", In IEEE Proceedings of the International Conference on Image Processing, Thessaloniki, Greece, 7-10 October.

8) Liang, K. M., Scott, S. D., and Waqas, M., 2004, "Detecting Pornographic Images", In Asian Conference on Computer Vision, Accv, Korea, pp. 497-502,

9) Gasparini, F., Corchs, S. and Schettini, R., 2005, "Pixel Based Skin Colour Classification Exploiting Explicit Skin Cluster Definition Methods", In Proceedings of International Colour Association (AIC) Colour, Granda, Spain, pp. 543-546, 8-13 May.

10) Lee, J. Y. and Yoo, S. I., 2002, "An Elliptical Boundary Model for Skin Color Detection", In Proceedings of the International Conference on Imaging Science, Systems, and Technology, Las Vegas, USA, 23-26 June.

11) Vezhnevets, V., Sazonov, V. and Andreeva, A., 2003, "A Survey Pixel-Based Color Detection Techniques", Graphicon, MoscowRussia, September.

12) Tretyakov, K., 2004, "Machine Learning Techniques in Spam Filtering", Data Mining Problem, MTAT, Vol. 3. 
13) Chai, D., Bouzerdoum A., 2000, "A Bayesian Approach to Skin Color Classification in $\mathrm{YC}$ bCr Color Space", IEEE Region Ten Conference (TENCON'2000), Kualu Lumpur, Malaysia, vol. II, pp. 421-424, 24-27 September.

14) Arentz, W. A., Olstad, B., 2004, "Classifying Offensive Sites Based on Image Content", Computer Vision and Image Understanding, Vol. 94, pp. 295-310.

15) Bosson, A., Cawley, G. C., Chan, Y. and Harvey, R., 2002, "NonRetrieval: Blocking Pornographic Images", In Proceeding International Conference on the Challenge of Image and Video Retrieval (CIVR), UK, London, pp. 50-60, 18-19 July.

16) Androutsopoulos, I., Paliouras, G., Karkaletsis, V. and Sakkis, G., 2000, "Learning to Filter Spam E-Mail: A Comparison of a Naive Bayesian and a Memory-Based Approach", Proceedings of the Workshop on Machine Learning and Textual Information Access, 4th European Conference on Principles and Practice of Knowledge Discovery in Database (PKDD), Lyon, France, 13-16 September.

17) Androutsopoulos, I., Koutsias, J. Chandrinos, K. V. and Spyropoulos, C. D., 2000, "An Experimental Comparison of Naive Bayesian and Keyword-Based Anti-Spam filtering with Personal Email Messages", In Proceeding of the $23^{\text {rd }}$ annual international (ACM SIGIR) Conference Research and Development in Information Retrieval, Athens, Greece, pp.160-167, 24-28 July.

18) Androutsopoulos, I., Koutsias, J., Chandrinos, K. V., Paliouras, G. and Spyropoulos, C. D., 2000, "An Evaluation of Naive Bayesian Anti-Spam Filtering", In Proceedings of the Workshop on Machine Learning in the New Information Age, 11th European Conference on Machine Learning, Barcelona, Catalonia, Spain, pp. 9-17, 31 May 2 June.

19) Forsyth, D. A., and Fleck, M. M., 1999, "Automatic Detection of Human Nudes", International Journal of Computer Vision, No.32 (1), August .

20) Chan, Y., Harvey, R. and Smith, D., 1999, "Building Systems to Block Pornography", In 2nd UK Conference on Image Retrieval: The Challenge of Image Retrieval (CIR'99), BCSEI Electronic Workshops in Computing Series, New Castle, UK, pp. 34-40, 25-26 February.

21) Secker, A., Freitas, A. A. and Timmis, J., 2003, "AISEC: an Artificial Immune System for E-mail Classification", In Proceeding of the Congress on Evolutionary Computation, Canberra, Australia, pp. 131-139, 8-12 December.

22) Umbaugh, E. S., 1998, "Computer Vision and Image Processing", Prentice Hall.

23) Fawcett, T., 2003, "ROC Graphics: Notes and practical Consideration for Researchers", Technical Report HPL, Hp Lab, Palo Alto, California, USA. 